\title{
FUTURO Y PERSPECTIVAS DEL TRABAJO EN EL MARCO DEL COVID-19
}

Future and perspectives of work in the framework of COVID-19

Carlos García Salas ${ }^{1}$

Recibido: 30 de abril de 2020 - Aceptado: 18 de mayo de 2020

\section{RESUMEN}

La pandemia que azota a la humanidad ha desnudado la desigualdad existente en todos los rincones del planeta y también la vulnerabilidad de la economía de mercado, provocando una recesión mundial calculada en 5,2\%, disminuyendo el ingreso per cápita en 3,7\%, empujando a la miseria a la mayoría de la sociedad. En este escenario el trabajo se ha catapultado como principal protagonista de esta tragedia, acechado por dos fenómenos: uno imprevisible, que es la pandemia misma, que ha ocasionado la pérdida de 400 millones de puestos de trabajo; y otro previsible, que es el avance de la tecnología en el mercado de trabajo que, así como ha creado, también ha destruido muchos puestos de trabajo. En este contexto, la Organización Internacional del Trabajo (OIT) está jugando un papel determinante, instando a los gobiernos al dialogo social y alertando acerca de los peligros de la automatización en el trabajo. Sobre esas realidades, Latinoamérica y en especial Colombia tienen que empezar a insertarse en el exigente contexto actual del mercado de trabajo mundial.

Palabras clave: Trabajo; Covid-19; Pandemia; Derecho laboral.

\begin{abstract}
The pandemic that plagues humanity has exposed the inequality that exists in all corners of the planet and also the vulnerability of the market economy, causing a global recession calculated at 5.2\%, and reducing per capita income by $3.7 \%$, pushing most of society into misery. In this scenario, work has been catapulted as the main protagonist of this tragedy, stalked by two phenomena: an unpredictable one, which is the pandemic itself, which has caused the loss of 400 million jobs; and another foreseeable one, which is the advancement of technology in the labor market that, as it has created, has also destroyed many jobs. In this context, the International Labor Organization (ILO) is playing a decisive role, urging governments to social dialogue and warning about the dangers of automation at work. On these realities, Latin America and especially Colombia have to start inserting themselves into the current demanding context of the world labor market.
\end{abstract}

\footnotetext{
1 Abogado, Magister en Derecho, profesor universitario y Magistrado de la Sala Laboral del Tribunal Superior del Distrito Judicial de Bolívar. Orcid: https://orcid.org/0000-0002-0999-8159 E-mail: cfgs55@ @otmail.com
} 
Keywords: Work; Covid-19; pandemic; labor law.

\section{INTRODUCCIÓN}

La pandemia que azota a la humanidad, ocasionada por el Covid-19, ha desnudado el grado de desigualdad social subyacente en todos los rincones del planeta y, a su vez, ha evidenciado la vulnerabilidad de las instituciones políticas y económicas. Lo anterior es de tal magnitud que los analistas han anunciado un periodo de recesión que el Fondo Monetario Internacional (FMI) ha denominado "El Gran Confinamiento" y que para la Unión Europea significaría una contracción económica del 71/2\% (Comisión Europea, 2020), mientras que para China la contracción del primer trimestre fue de $6,8 \%$.

Sin duda, esta recesión está impactando directa y negativamente en el mundo del trabajo, lo cual se traduce en una enorme pérdida de puestos de trabajo a nivel global. Al respecto, la Organización Internacional del Trabajo (OIT, 2020), en su informe sobre el Covid-19 y su influencia en el ámbito laboral global, calcula 305 millones de empleos perdidos para el segundo trimestre del presente año.

Por otra parte, son desalentadoras las imágenes que muestran a millares de personas haciendo filas para recibir los subsidios de desempleo en Estados Unidos, donde la recesión llegó a 4,8 (Forbes, 2020) y se calcula que el total de pérdida de empleos llegue a los 30 millones (El Tiempo, 2020); mientras que, en Colombia, solo en el mes de abril se perdieron 5,4 millones de empleos (El Tiempo, 2020).

Ante este panorama, resulta importante comprender que el trabajo está afrontando un desafío histórico, pues se ha constituido en el epicentro de dos circunstancias; a saber, una imprevisible, que es la actual pandemia, y otra previsible, que es el desarrollo tecnológico.

\section{El trabajo en la post pandemia}

La pandemia actual, además de afectar millones de puestos de trabajo, ha generado, según la OIT (OIT, 2020), un fenómeno muy evidente que es la profundización de la modalidad de teletrabajo, lo cual, ante la magnitud de la crisis, resulta ser un factor positivo, pero que no da lugar a nuevos puestos o cargos de trabajo.

De esa manera, esta nueva realidad, es decir, el teletrabajo, nos ubica frente a cambios de paradigmas en las organizaciones empresariales, como la flexibilización del trabajo, donde se ha impuesto, de forma inexorable, el trabajo remoto como el home office, el freelance, el trabajo por objetivos, entre otros. Sumado a ello, se han implantado protocolos de bioseguridad, distanciamiento social, limitación de viajes, paseos laborales, congresos, flexibilización de horarios de trabajo y de transporte. Sin lugar a duda, se irá consolidando lo que los analistas denominan la Gig Economy, esto es, la nueva economía del trabajo móvil, remoto, a demanda e independiente y la pluriactividad (En Naranja, 2019).

Por todo lo expuesto, en este momento resulta esencial reflexionar frente a la siguiente interrogante: ¿Se recuperarán los 305 millones de puestos de trabajo que según la OIT se habrán perdido en el segundo trimestre de este año? 
Realmente esto significaría una catástrofe humanitaria a la cual es imposible aplicarle la ley de conservación de la materia o ley de Lomonósov-Lavoisier (La ley de la conservación de la materia, es también llamada ley de conservación de la masa o Ley de Lomonósov-Lavoisier, en honor a sus creadores. Postula que la cantidad de materia antes y después de una transformación es siempre la misma), según la cual la materia no desaparece, sino que se transforma.

Pretender que esa cifra de destrucción de puestos de trabajo se recupere bajo nuevas modalidades de trabajo (teletrabajo, home office, freelance, trabajo remoto, cuentapropismo), sería desconocer el grado de dificultad económica que están afrontando miles de pequeñas y medianas empresas (pymes) propensas a desaparecer, y grandes empresas, como Hertz y Avianca, las cuales, como último recurso se han acogido a la ley de quiebra o bancarrota en Estados Unidos (Dinero, 2020).

Asimismo, tendríamos que analizar la situación de los países en vías de desarrollo, donde no existen esos gigantes empresariales, sino que se encuentran las pymes y que, por ejemplo, en Colombia aportan el 35\% del PIB y emplean alrededor de 16 millones de personas (Portafolio, 2019); empleos de los cuales, como se dijo, se han perdido 5,4 millones hasta el mes de abril en lo que va de confinamiento (El Tiempo, 2020); es decir, casi el $30 \%$ del personal de estas empresas. Como consecuencia de ello, el Estado adoptó medidas como el subsidio a la nómina y a la prima de servicio, según lo cual se pagará durante tres meses únicamente el $40 \%$ de un salario mínimo, para los empleados de todas las empresas que hayan registrado una disminución del $20 \%$ o más en su facturación (La República, 2020).

Entonces, teniendo en cuenta el grado de dificultad económica de cada país o región y el tiempo de recuperación de la crisis recesiva, no es seguro que se restablezca ni siquiera el $50 \%$ de los 305 millones de puestos de trabajo que están por perderse, y que para América latina la pandemia tendrá repercusiones devastadoras para el mercado laboral, exacerbando la desigualdad en la región, según reporta el Banco Interamericano de Desarrollo - BID (Portafolio, 2020).

Es por ello que la OIT (2020) propone la adopción de soluciones políticas urgentes y significativas para proteger tanto a las empresas, en particular a las más pequeñas, como a los trabajadores, sobre todo aquellos que operan en la economía informal.

En ese orden de ideas, son realmente preocupantes las proyecciones catastróficas sobre pérdida de empleo, puesto que, como lo afirma el análisis de la OIT (2020), se agranda la brecha de desigualdad. Esto último, en tanto que esta crisis golpea más fuerte a los sectores vulnerables del mercado de trabajo como son los 1600 millones de trabajadores informales. Además, la tasa de pobreza aumentará al menos 34 puntos porcentuales para los trabajadores informales en el mundo (OIT, 2020).

Bajo dicho contexto y como medidas de choque en esta época de recesión, la presencia del Estado en la dirección de la economía se hace más urgente que nunca, para incrementar el 
gasto público a través de inversiones en infraestructura, asistencia social, estimulación del empleo; es decir, recurrir a la clásica teoría keynesiana que en este caso resulta fundamental. Así, por ejemplo, el Gobierno colombiano aprobó el subsidio a la nómina en una cuantía de 6 billones de pesos (Morales, 2020); la Unión Europea autorizó la suma de 540 millones de euros como apoyo al empleo y a las empresas de los Estados miembros (Comisión Europea, 2020), y el Congreso de Estados Unidos avaló la suma de 2 billones de dólares para la lucha contra la pandemia, incluyendo 321 mil millones de dólares para apoyar a las pequeñas empresas (BBC News Mundo, 2020).

En consecuencia, será muy difícil y muy traumático para el mercado laboral salir indemne de esta crisis si el diálogo social a que exhorta la OIT no se implementa como política gubernamental (OIT, 2020), teniendo en cuenta que los sectores más vulnerables del mercado laboral son los trabajadores informales, que sobre pasan los 1600 millones en el mundo, según este organismo, y de los cuales, además, Colombia aporta 12,3 millones (Portafolio, 2020).

\section{El desarrollo tecnológico y el trabajo}

La inteligencia artificial (IA), la digitalización y la robótica, le agregan al análisis sobre el futuro del trabajo una gran dosis de expectativa y especulación sobre la posibilidad de que se reduzcan drásticamente las oportunidades de trabajo o, por el contrario, que estas se incrementen.

Al respecto, la Comisión Mundial sobre Futuro del Trabajo, creada por la OIT, en su informe de enero de 2019 (OIT, 2019), abordó el impacto de la tecnología en el trabajo y exhortó al uso de la tecnología para el trabajo decente y bajo el control humano. Lo anterior, considerando que la tecnología puede liberar a los trabajadores del trabajo arduo, la monotonía y la penuria; pero, a la vez, la automatización puede mermar la capacidad de control y autonomía de los trabajadores.

Por otra parte, se planteó que la inteligencia artificial debe estar bajo pleno control humano, para que garantice que las decisiones finales que afecten el trabajo sean asumidas por seres humanos y no por algoritmos o sensores que perjudiquen la dignidad del trabajador, pues estando Colombia entre los países más corruptos del mundo, se debe dejar claro que el trabajo no es una mercancía, ni el trabajador es un robot.

Ante el innegable avance de las plataformas digitales, dicha Comisión hizo énfasis en un sistema de gobernanza internacional para estas formas de trabajo que propendan por el respeto de los derechos mínimos laborales. Finalmente, la Comisión expuso su preocupación por la cantidad de datos que manejarán estas plataformas, puesto que esto significa un riesgo de violación de la intimidad de los trabajadores y una mayor discriminación laboral.

Esta preocupación de la OIT está en la misma dirección de lo expresado por Nick Bostrom, director del Instituto para el Futuro de la Humanidad, autor del libro "Superinteligencia", quien con su grupo de estudio llegó a la conclusión que las maquinas superinteligentes 
pronto tendrán la capacidad de pensar por sí solas; recomendando tomar precauciones para evitar la destrucción de la humanidad en manos de esta nueva fuente de vida inteligente (Opphenheimer, 2018).

Desde dicha perspectiva, la OIT (OIT, 2020) es optimista al visualizar las novedosas oportunidades de empleo que traerá la aplicación de nuevas tecnologías, incluso vaticina que la ecologización de la economía traerá más puestos de trabajo. En ese sentido, solo aboga por un trabajo decente y por la implementación del diálogo social como instrumento de concertación entre empleadores, trabajadores y Estados.

Así pues, lo cierto es que la tecnología afectará al mundo laboral de diferentes formas, atendiendo el grado de desarrollo tecnológico y científico de cada país o región. Por su parte, la OIT (OIT, 2020) reconoce que en dicha transición son muchos los que van a perder sus trabajos, y podrían ser los menos preparados para aprovechar las nuevas oportunidades. Adicional a ello, las competencias de hoy no se ajustarán a los trabajos de mañana, y las nuevas competencias adquiridas pueden quedar desfasadas rápidamente. Por ello, si dejamos que la economía de plataforma siga sin control como hasta ahora, surgirán generaciones de "jornaleros digitales" en pleno siglo XXI (OIT, 2020).

Teniendo en cuenta lo anterior, los datos actuales hablan de la pérdida de empleos a causa de la automatización. Por ejemplo, según un estudio de la Organización para la Cooperación y el Desarrollo Económicos (OCDE), “4,2 millones de españoles tienen más de un $70 \%$ de posibilidades de perder su empleo [...] a causa de los robots y la digitalización" (Luengo, 2020, p.1).

Según un estudio de Oxford Economics (Oxford Economics, 2019), para el año 2030, más de 1.5 millones de empleos se habrán perdido por causa de los robots en los Estados Unidos; mientas que, en China, se espera que ese número supere los 11 millones. Asimismo, en todos los Estados miembros de la Unión Europea, casi 2 millones de personas perderían el empleo debido a la automatización, de acuerdo con el informe. Los robots podrían asumir más de 20 millones de empleos de fabricación en todo el mundo para 2030 (Oxford Economics, 2019). "Según un nuevo estudio de Oxford Economics, en los próximos 11 años podría haber 14 millones de robots puestos a trabajar solo en China” (Delgado, 2020, p.1).

"Hace casi un año, un estudio de McKinsey Global Institute (MGI), con la participación de expertos del Departamento de Economía de Oxford y el Banco Mundial, pronosticó que entre 400 y 800 millones de personas serán desplazadas de sus puestos de trabajo en 2030 debido a la automatización.

'El Foro Económico Mundial (WEF) acaba de publicar su informe 'Future of Jobs 2018' [...]. Debido a los avances tecnológicos, en 2025 esta cifra significaría la pérdida de 75 millones de empleos. Pero sugiere que esta misma tecnología dará vida a más de 133 millones de nuevos roles de trabajo, con lo que se generarán 58 millones de nuevos empleos. [...] Se calcula que el 54\% de todos los empleados necesitarán formación en nuevas habilidades durante los próximos cinco años, se 
trataría de capacitación centrada en internet móvil de alta velocidad, inteligencia artificial, análisis de big data y cómputo en la nube, con lo que se impulsaría la demanda de un nuevo tipo de fuerza de trabajo que conviviría con la llegada de las máquinas. De hecho, el informe menciona que los humanos deberán adaptarse a este escenario al exigir mayor preparación, algo que sería un trabajo conjunto entre empresas, gobierno y la misma fuerza de trabajo". (Álvarez, 2018, pág. 1).

Para Latinoamérica, de acuerdo con el informe de la Comisión Económica para América Latina y el Caribe (CEPAL, 2019), el panorama es un tanto complejo, debido a la estructura del empleo y al rezago en la introducción de la tecnología. No obstante, reconoce que aplicando el método original propuesto por Frey y Osborne (Frey y Osborne, 2013), un $62 \%$ de los ocupados están con riesgo de sustitución tecnológica. Otros estudios señalan que para el 2030, a través de la implementación de la tecnología se eliminarán en Latinoamérica alrededor de 3,38 millones de empleos, según datos de la OCDE y la CEPAL (Dirksen, 2019).

Por otra parte, la especulación acerca de los efectos de la tecnología en el mercado de trabajo, ha llevado a que los analistas hablen de tecno-escépticos y tecno-optimistas, según se predique la destrucción o la creación de puestos de trabajo (Oppenheimer, 2018). Asimismo, personajes decididamente influyentes en el mundo de la informática como Bill Gates (Microsoft), Mark Zuckerberg (Facebook), Larry Page (Google) y Elon Musk (Telsa), consideran que la digitalización eliminará la mayoría de los trabajos existentes, sin posibilidad de que se creen nuevos trabajos en la misma cantidad ni con la misma velocidad (Gnutti, 2017).

En contraste, uno de los más grandes pensadores de esta centuria, el sociólogo y filósofo polaco británico Zygmund Bauman (Gnutti, 2017), considera que, ante el avance inevitable de la globalización y la tecnología, la humanidad afrontará un periodo de desocupación, por lo que ahora al desempleo se le denomina "Redundancia", todo ello merced a que la inteligencia artificial irá reemplazando al trabajo humano de manera inexorable. Por ello, el eminente filósofo sentencia categóricamente que hay que romper la conexión de empleo con el derecho a subsistir; es decir, vivir para ser libres, para lo cual es partidario de la renta básica universal; además, considera que los Estados modernos no pueden garantizar el pleno empleo que en estos momentos es una quimera.

\section{CONCLUSIONES}

Superada la recesión mundial provocada por la pandemia, pero sin haberse recuperado los 305 millones de puestos de trabajo perdidos, a nuestro protagonista (el trabajo) le queda el reto de afrontar el impacto tecnológico, que puede ser muy doloroso y brutal para los países periféricos, precisamente por el atraso científico y tecnológico endémico, aunado a la corrupción en que se sustentan, siendo Colombia, sino el primero, uno de los más corruptos del mundo según U.S.NEWS, donde se pierden 50 billones de pesos anualmente, según la contraloría (Torres, 2020), por lo que se hace necesario implementar, además del dialogo social que recomienda la OIT, rigurosas políticas de choque, no solo en el plano económico a través de la inversión social que genere empleo decente, sino mediante un vuelco en las políticas educativas, a fin de que se compadezcan con las tendencias mundiales del mercado

\footnotetext{
REVISTA Mario Alario

JURÍDICA D'Filippo
} 
de trabajo y para que le brinde a las generaciones venidera la posibilidad de insertarse en ese mundo de acuerdo a la oferta mundial, que va estar signada por la inteligencia artificial y la digitalización. Según el Banco Mundial (El Tiempo, 2020), Colombia destina el 4.5\% del PIB a la educación, (44.1 billones para el 2020), encontrándose por debajo de países como Cuba (12,8\%), Costa Rica (7,4\%); Bolivia (7,3\%); Venezuela (6.9\%); Brasil (6.2\%); Argentina (5.5\%); Chile (5.4\%); Ecuador (5.0\%); México(4.9\%); y Uruguay(4.9\%). Sin embargo, la inversión que hace Colombia se encuentra dentro del promedio del rango mundial de aportes a la educación, que fue de $4.487 \%$ del PIB para el 2018, según la UNESCO (Banco Mundial, 2020).

Y es que la OCDE en el análisis sobre Colombia en el documento "Políticas Prioritarias para un Desarrollo Inclusivo" de enero 2015, reconoce que nuestro país es uno de los países de Latinoamérica y del mundo con mayores desigualdades debido al elevado desempleo y al alto grado de informalidad laboral, por ello recomienda mejorar la calidad de la educación preescolar, básica y terciaria, haciéndola más inclusiva, centrándose en los conocimientos que exige el mercado laboral, porque los programas de educación actual no llenan esas necesidades, ya que según el estudio los CERES (Los Centros Regionales de Educación Superior son una estrategia del Ministerio de Educación Nacional que busca desconcentrar la oferta en educación superior (hoy centrada en las ciudades) y ampliar su cobertura; con el objetivo de hacer más equitativo el acceso y contribuir al desarrollo social y económico de las comunidades) y el SENA (El Servicio Nacional de Aprendizaje es un establecimiento público de orden nacional de Colombia, con personería jurídica, patrimonio propio e independiente, y autonomía administrativa; adscrito al Ministerio del Trabajo. Ofrece formación gratuita a millones de colombianos, con programas técnicos, tecnológicos y complementarios, enfocados en el desarrollo económico, tecnológico y social del país) incluyen tecnologías anticuadas y formación de dudosa calidad, y sobre el SENA manifiesta que sobre sus programas técnicos no se tienen datos acerca de la calidad de sus docentes y el desempeño de sus egresados en el mercado laboral (OCDE, 2015).

No podemos resignarnos a seguir siendo meros exportadores de materias primas sin valor agregado. Mucho menos a convertirnos en bodega de reciclaje del trabajo informal, o en la reserva de mano de obra poco calificada de la que se nutren los países desarrollados.

Ante este panorama, nuestro sistema educativo debe ser más inclusivo, estando obligado a democratizarse, facilitando el acceso a la población desfavorecida, haciéndola gratuita, y tomando como centro al ser humano, aunque parezca redundante y, además, nuestra educación debe hacer énfasis en:

- Ciencias humanas.

- Matemáticas (Colombia tiene 2.9 años de atraso de rendimiento escolar con relación a la media de la OCDE).

- Educación bilingüe con énfasis en el idioma inglés.

- Carreras científicas, técnicas o tecnológicas, con énfasis en internet móvil de alta velocidad, inteligencia artificial, análisis de big data y cómputo en la nube. 
- Abordar el deporte como disciplina científica que permita formar deportistas de alto rendimiento con carácter competitivo.

- Estudios medioambientales.

"Si Colombia quiere situarse como la nación con mejor formación de América

Latina en 2025, deberá priorizar las reformas que ofrezcan una mejora clara de los resultados de aprendizaje: una buena estrategia de formación en

educación preescolar, más calidad de la dirección de los centros educativos y del profesorado, y vínculos más sólidos con el mercado laboral” (OCDE,

\section{5, p. 23)}

\section{REFERENCIAS BIBLIOGRÁFICAS}

Álvarez, R. (2018). La automatización eliminará 75 millones de empleos para 2025, pero creará 133 millones de nuevas funciones, según WEF. En Portal Xataka. Publicado el 22 de septiembre de 2018. Recuperado de: https://www.xataka.com/robotica-eia/automatizacion-eliminara-75-millones-empleos-para-2025-creara-133-millonesnuevas-funciones-wef

Banco Mundial. (2020). Gasto público en educación, total (\% del PIB). Recuperado de: https://datos.bancomundial.org/indicador/SE.XPD.TOTL.GD.ZS

BBC News Mundo. (2020). Coronavirus | EE.UU. aprueba un paquete de ayudas económicas de US\$2 billones, el más grande de su historia. Recuperado de: https://www.bbc.com/mundo/noticias-internacional-52045106

Bostrom, Nick. (2014). Superintelligence: Paths, Dangers, Strategies. Oxford, Reino Unido: Universidad de Oxford.

Comisión Europea. (2020). Empleo y economía durante la pandemia de coronavirus. Recuperado de: https://ec.europa.eu/info/live-work-travel-eu/health/coronavirusresponse/jobs-and-economy-during-coronavirus-pandemic es

Comisión Europea. (2020). Respuesta común de la UE a la COVID-19. Recuperado de: https://europa.eu/european-union/coronavirus-response es

Departamento Administrativo Nacional de Estadística [DANE]. (2020). Información abril 2020. Recuperado de: https://www.dane.gov.co/index.php/estadisticas-portema/mercado-laboral/empleo-y-desempleo

Dinero. (2020). ¿Cuál es el nivel de desempleo que deja el coronavirus en Colombia? 14 de abril de 2020. Recuperado de: https://www.dinero.com/pais/articulo/cual-es-el-nivelde-desempleo-que-deja-el-coronavirus-en-colombia/286216 
Dinero. (2020). El confinamiento por la covid-19 lleva a Hertz a la bancarrota en EEUU y Canadá. 23 de mayo de 2020. Recuperado de: https://www.dinero.com/empresas/articulo/hertz-se-acoge-a-ley-de-quiebras-enestados-unidos-y-canada-por-la-covid-19/286636

Dirksen, U. (2019). Trabajo del futuro y futuro del trabajo. En Nueva Sociedad. Núm. 279, enero-febrero 2019. Recuperado de: https://nuso.org/articulo/trabajo-del-futuro-yfuturo-del-trabajo/

El Tiempo. (2020). EE. UU. destruirá alrededor de 30 millones de empleos. 23 de abril de 2020. Recuperado de: https://www.eltiempo.com/mundo/eeuu-y-canada/cifra-dedesempleo-en-ee-uu-durante-crisis-del-coronavirus-487678

En Naranja. (2019). ¿Qué es la gig economy? 22 de agosto de 2019. Recuperado de: https://www.ennaranja.com/para-ahorradores/que-es-la-gig-economy/

Forbes. (2020). Economía de EEUU se contrae 4,8\% en primer trimestre. 29 de abril de 2020. Recuperado de: https://forbes.co/2020/04/29/economia-y-finanzas/economia-deeeuu-se-contrae-48-en-primer-trimestre/

Frey, C. y Osborne, M. (2013). El futuro del empleo: ¿Cuán susceptibles son los empleos ante la computarización? Oxford, Reino Unido: Universidad de Oxford.

Gnutti, R. (2017). El mundo sin trabajo. Pensando con Zygmunt Bauman. Barcelona, España: Icaria.

Kalach Torres, Gina María. (2016). Las comisiones de la verdad en Colombia. En Revista Jurídica Mario Alario D'Filippo, vol. 8, núm. 16, p. 106-124 Recuperado de: https://doi.org/10.32997/2256-2796-vol.8-num.16-2016-1534

La República. (2020). Gobierno publicó decreto para el subsidio de la nómina de empleados. 9 de mayo 2020. Recuperado de: https://www.larepublica.co/economia/gobierno-publico-decreto-para-el-subsidio-de-lanomina-de-empleados-3003987

Luengo, C. (2020). Cuando la tecnología y el empleo están reñidos: el efecto de los robots en los puestos de trabajo. En Computer Hoy, 1 de febrero de 2020. Recuperado de: https://computerhoy.com/reportajes/industria/cuando-tecnologia-empleo-estan-renidosefecto-robots-puestos-trabajo-569567

Morales, M. (2020). Aporte estatal para el pago de nóminas de empresas, a prueba de fraude. En El Tiempo, 9 de mayo de 2020. Recuperado de: https://www.eltiempo.com/economia/sectores/claves-del-decreto-con-el-que-elgobierno-ayudara-a-pagar-nominas-de-empresas-493556

Oppenheimer, A. (2018). Sálvese quien pueda. El futuro del trabajo en la era de la automatización. Madrid, España: Debate. 
Organización de las Naciones Unidas. Comisión Económica para América Latina y el Caribe (CEPAL). (2019). Cambio tecnológico y empleo: una perspectiva latinoamericana. Riesgos de la sustitución tecnológica del trabajo humano y desafíos de la generación de nuevos puestos de trabajo. Recuperado de: https://www.cepal.org/es/publicaciones/44637-cambio-tecnologico-empleoperspectiva-latinoamericana-riesgos-la-sustitucion

Organización de las Naciones Unidas. Comisión Económica para América Latina y el Caribe (CEPAL). (2020). CEPAL propone avanzar hacia un ingreso básico para ayudar a la población más vulnerable a superar los efectos del coronavirus. Recuperado de: https://www.cepal.org/es/comunicados/cepal-propone-avanzar-un-ingreso-basicoayudar-la-poblacion-mas-vulnerable-superar

Organización Internacional del Trabajo (OIT). Comisión Mundial sobre el futuro del trabajo. (2019). Informe: Trabajar para un futuro más prometedor. 22 de enero de 2019. Recuperado de: https://www.ilo.org/global/topics/future-ofwork/publications/WCMS_662442/lang--es/index.htm https://www.ilo.org/global/topics/future-of-work/publications/WCMS 662442/lang-es/index.htm

Organización Internacional del Trabajo (OIT). (2020). Informe: Panorama laboral 2019. América Latina y el Caribe. 28 de enero de 2020. Recuperado de: https://www.ilo.org/americas/publicaciones/WCMS_732198/lang--es/index.htm

Organización Internacional del Trabajo (OIT). (2020). Observatorio de la OIT: El COVID19 y el mundo del trabajo. Tercera edición. Estimaciones actualizadas y análisis. 29 de abril de 2020. Recuperado de: https://www.ilo.org/wcmsp5/groups/public/---dgreports/--dcomm/documents/briefingnote/wcms_743154.pdf

Organización para la Cooperación y el Desarrollo Económico (OCDE). (2015). Serie "Mejores Políticas". Colombia: políticas prioritarias para un desarrollo inclusivo. Enero 2015. Recuperado de: https://www.oecd.org/about/publishing/colombia-politicasprioritarias-para-un-desarrollo-inclusivo.pdf

Oxford Economics. (2019). How robots change the world. Junio 2019. Recuperado de: http://resources.oxfordeconomics.com/how-robots-change-theworld\#: :text=The $\% 20$ far $\% 2$ Dreaching $\% 20$ results $\% 20$ will,two $\% 20$ decades $\% 2 \mathrm{C} \% 20 \mathrm{t}$ o\%202.25\%20million.\&text $=$ The $\% 20$ rise $\% 20$ of $\% 20$ the $\% 20$ robots $\% 20$ will $\% 20$ boost $\%$ 20productivity\%20and\%20economic\%20growth

Portafolio. (2019). Mipymes son la fuente de empleo de más de 16 millones de colombianos. 26 de septiembre de 2019. Recuperado de: https://www.portafolio.co/economia/mipymes-son-la-fuente-de-empleo-de-mas-de-16millones-de-colombianos-533962 
Portafolio. (2020). Los sectores con mayor riesgo de pérdida de empleo por el Covid- 19. 4 de mayo de 2020. Recuperado de: https://www.portafolio.co/economia/los-sectorescon-mayor-riesgo-de-perdida-de-empleo-por-el-covid-19-540483

Taylor, Chloe. (2019). Los robots podrían tomar más de 20 millones de empleos para 2030, según un estudio. En el blog: Economía y Tecnología en Trujillo. 27 de junio de 2019. Recuperado de: http://economiaytecnologiaentrujillo.blogspot.com/2019/06/los-robotspodrian-tomar-mas-de-20.html

Torres, M. (2020). Transparencia por Colombia critica ranking que puso a Colombia como el "país más corrupto". En RCN Radio, 17 de enero de 2020. Recuperado de: https://www.rcnradio.com/politica/transparencia-por-colombia-critica-ranking-quepuso-colombia-como-el-pais-mas-corrupto

Viguri Perea, A. y Chiara Marullo, M. (2016). El derecho a un medio ambiente sano y la encrucijada de los alimentos transgénicos. En Revista Jurídica Mario Alario D'Filippo, Vol. 8, Número 15: 100-111. Recuperado de: https://doi.org/10.32997/2256-2796-vol.8$\underline{\text { num. } 15-2016-1526}$

Suarez, Y., y De León, G. (2019). Inteligencia artificial y su aplicación en la administración de justicia. En Revista Jurídica Mario Alario D'Filippo, vol. 11, núm. 21, p. 71-83. Recuperado de: https://doi.org/10.32997/2256-2796-vol.11-num.21-2019-2501 